\title{
The Co-planarity and Symmetry Principle of Earthquake Occurrence
}

\author{
Zhe Yin ${ }^{1,2}$ \\ ${ }^{1}$ Department of Information Management, Beijing University, Beijing, China \\ ${ }^{2}$ Mathematics Department, Yanbian University, Yanji, China \\ E-mail:yinzhe@ybu.edu.cn \\ Received February 28, 2010; revised March 26, 2010; accepted April 20, 2010
}

\begin{abstract}
Under the assumption that the earth movement tendency is a sphere, the author does research about the earthquakes occurrence between 2001 and 2010 which over 6 scales of magnitude on occurred time (UTC), magnitude, longitude, latitude and other data, then give out the causal relationship of earthquakes and the co-planarity and symmetry theory of earthquake occurrence. Also, the author does empirical analysis in the paper.
\end{abstract}

Keywords: Earthquake, Co-planarity, Symmetry

\section{Introduction (Heading 1)}

Research on earthquakes is one of the most important elements about disaster prevention study, because of its devastating and destructive. Especially in recent years, with the rapid development of modern economic activity, destruction to the Earth was continuous. The frequency tends to rise and there are more large earthquakes. Therefore, how to analyze the reason of the earthquake correctly, and predict earthquakes effectively becomes a hot topic in nowadays.

Under the assumption that the earth movement tendency is a sphere and mountains fall to the surface of the earth; estrogens and basin rise to the surface of the Earth. This article gives out the co-planarity and symmetry theory of earthquake occurrence according to research about the earthquakes occurrence between 2001 and 2010 which over 6 scales of magnitude.

\section{Questions and Relevant Definitions}

The Earth, like a very sensitive being, according to the laws of nature, it has the desire to achieve a balance of features. In this process, it will show a particular trend.

As shown in Figure 1, we give some relevant definitions here:

Ecliptic axis: an axis through the center of the Earth which is a vertical line to the ecliptic plane.

Equatorial coordinate system: a celestial coordinate system of the equatorial plane and the axis of rotation.

Ecliptic system of coordinates: a celestial coordinate system of the ecliptic plane and ecliptic axis.

Tangent plane parallel to the equator: the Earth's equatorial plane parallel to the tangent plane (Tangent plane parallel the equator), shown as TPPTE.

Ecliptic line tangent plane: parallel to the ecliptic of the earth tangent plane (Ecliptic line tangent plane), shown as ELTP.

Equatorial vertical plane: after the Earth's surface

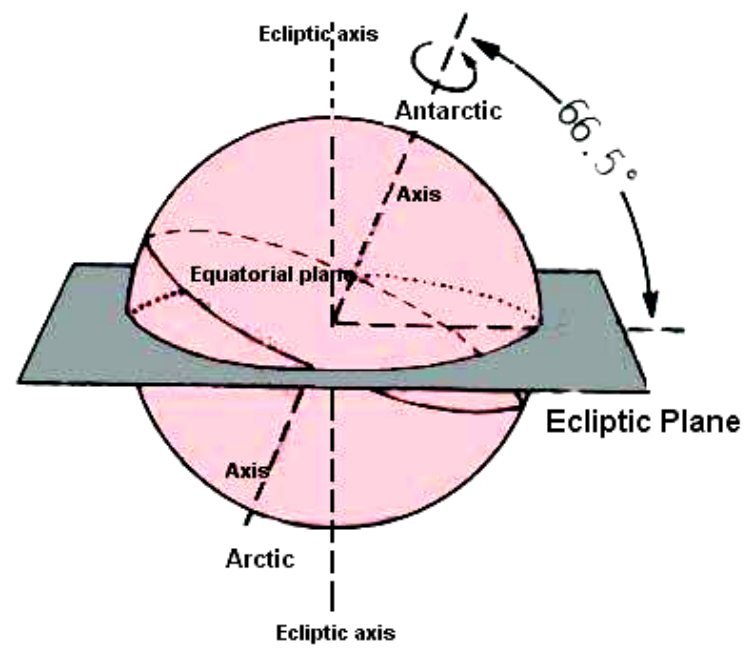

Figure 1. Plane figure. 
shock point and the spin axis of the tangent plane (Equatorial vertical plane), shown as EVP.

Longitudinal ecliptic plane: after a shock point and the yellow surface of the Earth axis tangent plane (Ecliptic plane vertical), shown as EPV.

Circular symmetry point: Circular, shown as CSP.

Similar point: Similar Point, shown as SP.

Earthquake effects: measure the Earth's surface; the intensity level of shock is unstable, the stability of the boundary tangent plane level. Plane is the ball away from the more deviation, the more poor results, and the more unstable and more likely point of this earthquake.

\section{Basic Assumptions and the Co-Planarity and Symmetry Principle of Earthquake}

\subsection{Basic Assumptions}

1) The Earth became positive sphere with earthquakes which rise or low the ground. That is, because of the ecliptic (23.26 degrees), the Ecliptic Plane TPPTE line tangent planes ELTP have tended to be round;

2) The Earth has tended to balance, and because the earthquake, to re-balance;

3) Crustal thickness tends to vary. Earth's core and mantle movement space tends to a ball space;

4) With the earthquake, the earth rotation axis offset. When there is a earthquake which makes the low rise, the earth rotation axis moves to the seismic vertical, those makes the high low is opposite.

\subsection{Co-planarity and Symmetry Principle}

After the observation of earthquakes on Earth N (154 earthquakes over 6 scales of magnitude in this article) times, it is occur time $\mathrm{H}_{\mathrm{K}}, \mathrm{H}_{\mathrm{K}+1}$ of adjacent earthquakes $\mathrm{M}_{\mathrm{K}}, \mathrm{M}_{\mathrm{K}+1}$ measure with UTC.

A) The previous earthquake is a cause of the after. The tangent plane balances was destroyed, then tend to a new balance, which Ecliptic line tangent plane generate new state plane all the times.

B) Seismic vertical $M_{K}$ and seismic vertical $M_{K+1}$ are on the same plane at the time $\mathrm{H}_{\mathrm{K}}$ or $\mathrm{H}_{\mathrm{K}+1}$. That is parallel to the cut-plane in the equatorial plane or Ecliptic line cut the ecliptic plane, or longitudinal vertical plane or equatorial plane.

C) If the tangent of the plane where seismic vertical $M_{K}$ and seismic vertical $M_{K+1}$ are tends to round, or fall-rise movement in the earthquake tends to stable, $\mathrm{M}_{\mathrm{K}}$ and $\mathrm{M}_{\mathrm{K}+1}$ tends to symmetry in the circle; otherwise, coplanar circle from the difference of the most unstable point will have an earthquake.
D) After earthquake on $M_{K}$ occurs, if the most unstable point (poor seismic effect) is still $\mathrm{M}_{\mathrm{K}}$, earthquake $\mathrm{M}_{\mathrm{K}+1}$ will take place near the same point.

\section{Research Basis and Calculation Method}

Assumptions (1), (2), (3) are prerequisite of conclusion (A), (B), (C) and (D); symmetry points including the spin axis symmetry, yellow axis symmetry and geocentric axial symmetry. With the existence of the ecliptic obliquity (ecliptic plane and the equatorial plane angle 23.26 degrees) and the Ecliptic plane tangent line, parallel to the equatorial plane tangent tends to a circle, the shape of the earth tends to a sphere becomes possible. This can be concluded with (C) and (D). Assumptions (4) decided the longitudinal movement of the $\mathrm{M}_{\mathrm{K}+1}$ after $\mathrm{M}_{\mathrm{K}}$. Assumptions (1) and (3) show the possibility that the earthquake and the earthquake potential basis for the order. Be noted that symmetrical points at any time change the fact that, in the premise revolution, a startled different points of the Ecliptic OK UTC timing of your cutting surface is subject to change, and cut parallel to the equatorial plane remain unchanged.

Earthquake time: the time the earthquake is very important, when the earthquake occurred; it generates and identifies Ecliptic line tangent plane, tangent plane parallel to the equator, the ecliptic plane and the equatorial vertical longitudinal cut tangent plane, as well as the corresponding point of the plane of symmetry at the same time.

Symmetrical points on the calculation: Earth autobiographical spherical coordinates on the equatorial plane symmetry coordinates of points on the simple calculation, the same latitude, longitude 180 degrees plus or minus 180 degrees. The following calculation Ecliptic coordinate system on the surface of the ball symmetric point computation of positions is shown in Figure 2 .

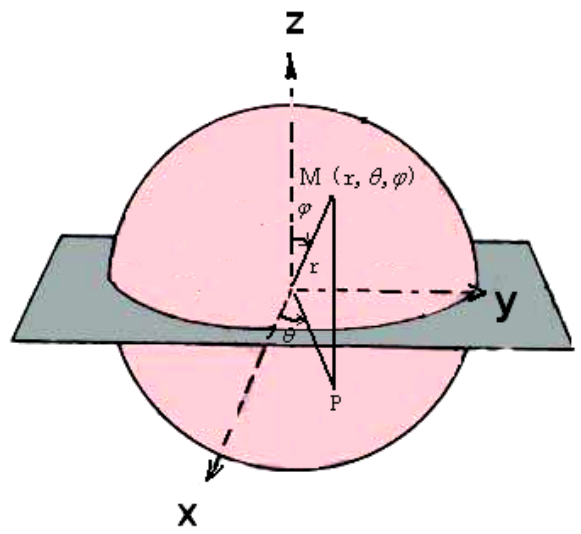

Figure 2. Caculations. 
Shock point set $\mathrm{M}$ of lat $=\mathrm{W}$, long $=\mathrm{J}$, time $($ UTC) $=\mathrm{a}$ : $\mathrm{b}$, then $\varphi_{0}=90-\mathrm{W}, \theta_{0}=\mathrm{J}+(\mathrm{a}+\mathrm{b} / 60) \times 15$

Step2:

$$
\begin{gathered}
\left\{\begin{array}{l}
x=\sin \varphi_{0} \cos \theta_{0} \\
y=\sin \varphi_{0} \sin \theta_{0} \\
z=\cos \varphi_{0}
\end{array}\right. \\
0^{\circ} \leq \theta_{0} \leq 360^{\circ}, 0^{\circ} \leq \varphi_{0} \leq 180^{\circ}
\end{gathered}
$$

Step3: To the counterclockwise rotation $23^{\circ} 26^{\prime}$, then

$$
\left\{\begin{array}{l}
x^{\prime}=x \cos 23^{\circ} 26^{\prime}+z \sin 23^{\circ} 26^{\prime} \\
y^{\prime}=y \\
z^{\prime}=x \sin 23^{\circ} 26^{\prime}-z \cos 23^{\circ} 26^{\prime}
\end{array}\right.
$$

$M$ point symmetry point assignment

Step4: Clockwise rotation to $23^{\circ} 26^{\prime}$, then

$$
\left\{\begin{array}{l}
x^{\prime \prime}=x^{\prime} \cos 23^{\circ} 26^{\prime}-z^{\prime} \sin 23^{\circ} 26^{\prime} \\
y^{\prime \prime}=y^{\prime} \\
z^{\prime \prime}=x^{\prime} \sin 23^{\circ} 26^{\prime}+z^{\prime} \cos 23^{\circ} 26^{\prime}
\end{array}\right.
$$

Step5

$$
\left\{\begin{array}{l}
x^{\prime \prime}=\sin \varphi_{1} \cos \theta_{1} \\
y^{\prime \prime}=\sin \varphi_{1} \sin \theta_{1} \\
z^{\prime \prime}=\cos \varphi_{1}
\end{array}\right.
$$

Step6: symmetry point, lat $=\mathrm{W}^{\prime}=90-\varphi_{1}$, long $=\theta_{1}$ $-(a+b / 60) \times 15$

\section{Results}

According to the information of 154 earthquakes over 6 scales of magnitude over the world from the USA Earthquake Observation Council, the causal relationship common area, symmetry of them can be concluded as following (Table 1) [1]: in the Comments, the next item TPPTE cut parallel to the earthquake in the equatorial plane, ELTP that the next seismic line cutting in the Ecliptic plane, EVP that the next earthquake in the vertical plane perpendicular to the equator, EPV that the next earthquake in the vertical longitudinal ecliptic plane. A total of circular symmetry point, then increasing the CSP, such as TPPTECSP; similar point with the SP said. Two adjacent seismic $\mathrm{M}_{\mathrm{K}}$ and $\mathrm{M}_{\mathrm{K}+1}$, at present, earthquake moment $\mathrm{H}_{\mathrm{K}}$, when the total surface, then added 1; when the time after the earthquake $\mathrm{H}_{\mathrm{K}+1}$, when the total surface, then added 2 .
Table 1. Original data.

2001

\begin{tabular}{ccccccc}
\hline Date & $\begin{array}{c}\text { Time } \\
\text { (UTC) }\end{array}$ & Place & Lat. & Long. Magnitude & Comments \\
\hline Jan 13 & $17: 33$ & Salvador & 13.04 & -88.66 & 7.7 & ELTPCSP1 \\
Jan 26 & $03: 16$ & India & 23.39 & 70.23 & 7.7 & TPPTECSP1
\end{tabular}

$\begin{array}{llllll}\text { Feb } 13 & 14: 22 & \text { Salvador } & 13.67 & -88.94 & 6.6\end{array}$

\begin{tabular}{|c|c|c|c|c|c|c|}
\hline Date & $\begin{array}{c}\text { Time } \\
\text { (UTC) }\end{array}$ & Place & Lat. & Long. & Magnitude & Comments \\
\hline Feb 3 & $07: 11$ & Turkey & 38.573 & 31.271 & 6.5 & TPPTE1 \\
\hline Mar 3 & $12: 08$ & $\begin{array}{l}\text { Afghani- } \\
\text { stan }\end{array}$ & 36.543 & 70.424 & 7.4 & SP1 \\
\hline Mar 25 & $14: 56$ & $\begin{array}{l}\text { Afghani- } \\
\text { stan }\end{array}$ & 36.06 & 69.32 & 6.1 & TPPTE1 \\
\hline Jun 22 & $02: 58$ & Iran & 35.63 & 49.05 & 6.5 & TPPTE1 \\
\hline Oct 31 & $10: 32$ & Italy & 41.79 & 14.87 & 5.9 & ELTPCSP1 \\
\hline Nov 3 & $22: 12$ & $\begin{array}{l}\text { Alaska, } \\
\text { USA }\end{array}$ & 63.52 & -147.4 & 7.9 & EPV1 \\
\hline
\end{tabular}

$\begin{array}{lllllll}\text { Feb } 28 & 18: 54 & \text { USA } & 47.11 & -122.6 & 6.8 & \text { EPV2 }\end{array}$

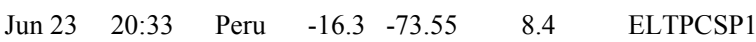

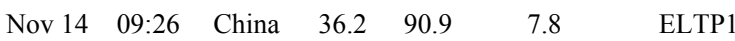

2002

2003

\begin{tabular}{cc}
\hline Time \\
(UTC)
\end{tabular} Place Lat. Long. $\begin{gathered}\text { Magni- } \\
\text { tude }\end{gathered}$ Comments

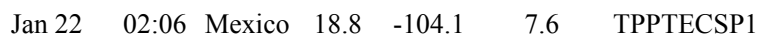

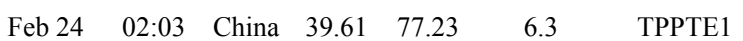

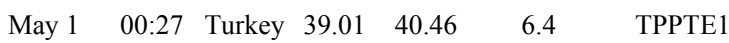




\begin{tabular}{|c|c|c|c|c|c|c|}
\hline May 21 & 18 & 44 Algeria 3 & 36.96 & 3.63 & 6.8 & TPPTE1 \\
\hline Sep 25 & 19 & 50 Japan 4 & $41.82 \quad 14$ & 43.91 & 8.3 & ELTP2 \\
\hline Nov 17 & $17 \quad 06$ & 43 USA 5 & $\begin{array}{ll}51.15 & 17\end{array}$ & 78.65 & 7.8 & \\
\hline Dec 22 & 19 & 15 USA 3 & $\begin{array}{ll}35.71 & -1\end{array}$ & 121.1 & 6.6 & ELTP2 \\
\hline \multicolumn{2}{|c|}{ Dec 26} & Iran & $29.00 \quad 5$ & 58.31 & 6.6 & ELTP1 \\
\hline \multicolumn{7}{|l|}{2004} \\
\hline Date & $\begin{array}{c}\text { Time } \\
\text { (UTC) }\end{array}$ & Place & Lat. & Long. & $\begin{array}{l}\text { Mag- } \\
\text { nitude }\end{array}$ & Comments \\
\hline Feb 5 & $21: 05$ & Indonesia & -3.61 & 135.53 & 7.0 & ELTP1 \\
\hline Feb 24 & $02: 27$ & Gibraltar & 35.14 & $\begin{array}{l}4 \\
4.99\end{array}$ & 6.4 & TPPTE1 \\
\hline May 28 & $12: 38$ & Iran & 36.29 & 51.61 & 6.3 & TPPTE1 \\
\hline Sep 5 & $10: 07$ & Japan & 33.07 & $7 \quad 136.62$ & 7.2 & SP1 \\
\hline Sep 5 & $14: 57$ & Japan & 33.14 & $4 \quad 137.07$ & 7.4 & ELTP1 \\
\hline Oct 9 & $21: 26$ & Nicaragua & 12 & -86 & 7.0 & ELTPCSP1 \\
\hline Oct 23 & $08: 56$ & Japan & 37.3 & 138.8 & 6.6 & EVP1 \\
\hline Nov 11 & $21: 26$ & East Timor & r $\quad-8.15$ & 124.87 & 7.5 & ELTPCSP1 \\
\hline Nov 15 & 09:06 & Colombia & 4.7 & -77.51 & 7.2 & ELTP1 \\
\hline Nov 20 & 08:07 & Costa Rica & 9.6 & -84.17 & 6.4 & TPPTE1 \\
\hline Nov 21 & $11: 31$ & Islands & 15.68 & -61.71 & 6.3 & TPPTECSP1 \\
\hline Nov 26 & $02: 25$ & Indonesia & -3.60 & 135.40 & 7.1 & EPV1 \\
\hline Nov 28 & $18: 32$ & Japan & 42.94 & 145.28 & 7.0 & EVP1 \\
\hline $\operatorname{Dec} 23$ & $14: 59$ & New Zealan & nd -50.24 & 4160.13 & 8.1 & ELTP1 \\
\hline Dec 26 & $00: 58$ & Indonesia & 3.30 & 95.87 & 9.1 & ELTP1 \\
\hline \multicolumn{7}{|l|}{2005} \\
\hline Date & $\begin{array}{c}\text { Time } \\
\text { (UTC) }\end{array}$ & Place & Lat. & Long. & $\begin{array}{c}\text { Magni- } \\
\text { tude }\end{array}$ & Comments \\
\hline Feb 22 & $02: 25$ & Iran & 30.726 & 56.817 & 6.4 & TPPTE1 \\
\hline Mar 20 & 01:53 & Japan & 33.54 & 130.12 & 6.6 & EPV1 \\
\hline Mar 28 & $16: 09$ & Indonesia & 2.08 & 97.11 & 8.6 & ELTPCSP1 \\
\hline Jun 13 & $22: 44$ & Chile & -19.89 & -69.12 & 7.8 & EPV1 \\
\hline Jun 15 & $02: 50$ & California & 41.284 & -125.9 & 7.2 & TPPTE1 \\
\hline Aug 16 & $02: 46$ & Japan & 38.259 & 148.98 & 7.2 & EPV1 \\
\hline Sep 9 & $07: 26$ & New Guinea & -4.539 & 153.47 & 7.6 & TPPTE1 \\
\hline Sep 26 & $01: 56$ & Peru & -5.68 & -76.4 & 7.5 & EPVD2 \\
\hline Oct 8 & $03: 50$ & Pakistan & 34.43 & 73.54 & 7.6 & TPPTE1 \\
\hline Nov 26 & 00:49 & China & 29.7 & 115.7 & 5.2 & TPPTE1 \\
\hline Nov 27 & $10: 22$ & Iran & 26.77 & 55.86 & 5.9 & EPV1 \\
\hline Dec 5 & $12: 19$ & Tanzania & -6.212 & 29.599 & 6.8 & EPV1 \\
\hline
\end{tabular}

2006

\begin{tabular}{ccccccc}
\hline Date & $\begin{array}{c}\text { Time } \\
\text { (UTC) }\end{array}$ & Place & Lat. & Long. & $\begin{array}{c}\text { Mag- } \\
\text { nitude }\end{array}$ & Comments \\
\hline Jan 8 & $11: 34$ & Greece & 36.250 & 23.498 & 6.7 & EPV1 \\
Feb 22 & $22: 19$ & $\begin{array}{c}\text { Mozam- } \\
\text { bique }\end{array}$ & -21.32 & 33.58 & 7.0 & EPV1 \\
Apr 20 & $23: 25$ & Russia & 61.075 & 167.08 & 7.6 & EPV1 \\
May 3 & $15: 26$ & Tonga & -20.13 & -174.1 & 8.0 & EVP1 \\
May 16 & $10: 39$ & New Zea- & -31.55 & -179.2 & 7.4 & ELTP2 \\
May 27 & $22: 54$ & Indonesia & 7.977 & 110.31 & 6.3 & EPV1 \\
Jul 17 & $08: 19$ & Indonesia & -9.334 & 107.26 & 7.7 & EPV1 \\
Aug 20 & $03: 41$ & Scotia Sea & -61.02 & -34.37 & 7.0 & EPV1 \\
Sep 10 & $14: 56$ & Mexico & 26.339 & -86.56 & 5.9 & TPPTE1 \\
Oct 15 & $17: 07$ & USA & 19.801 & -156.0 & 6.7 & EPV1 \\
Nov 15 & $11: 14$ & Russia & 46.616 & 153.22 & 8.3 & ELTP1 \\
Dec 26 & $12: 26$ & Taiwan & 21.818 & 120.53 & 7.1 & ELTP2 \\
\hline
\end{tabular}

2007

\begin{tabular}{|c|c|c|c|c|c|c|}
\hline Date & $\begin{array}{c}\text { Time } \\
\text { (UTC) }\end{array}$ & ace & at. & ong. & $\begin{array}{l}\text { Mag- } \\
\text { itude }\end{array}$ & $\mathrm{Ce}$ \\
\hline 13 & 5 & & & & & \\
\hline Jan 21 & $11: 27$ & Molucca Sea & 1.207 & 126.29 & & PV1 \\
\hline Feb 12 & $11: 27$ & Portugal & 36.09 & 0.26 & .0 & LTP2 \\
\hline Mar 6 & 05:49 & Indonesia & -0.490 & 100.52 & 4 & ELTP2 \\
\hline Mar 25 & $00: 40$ & Vanuatu & -20.59 & 169.41 & .1 & EPV1 \\
\hline Mar 25 & $00: 42$ & Japan & 37.537 & 136.43 & 6.7 & EPV1 \\
\hline Apr 1 & $20: 39$ & Islands & -8.474 & 156.95 & 8.1 & ELTP1 \\
\hline May 16 & 08:56 & Laos & 20.470 & 100.70 & 6.3 & ELTP1 \\
\hline Jun 2 & $21: 34$ & China & 23.013 & 101.05 & 6.1 & ELTPCSP1 \\
\hline Jun 13 & $19: 29$ & Guatemala & 13.628 & -90.73 & 6.7 & LTPCSP1 \\
\hline Jul 16 & 01:13 & 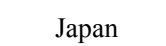 & 37.574 & 138.44 & 6 & קסד \\
\hline Jul 18 & $17: 30$ & & & & & \\
\hline Aug 1 & $17: 08$ & & -15.67 & 167.60 & & \\
\hline Aug 2 & $02: 37$ & Russia & 47.259 & 141.75 & 6.2 & EPV1 \\
\hline Aug 8 & $17: 04$ & Indonesia & -5.968 & 107.65 & 7.5 & ELTPCSP2 \\
\hline Aug 15 & $23: 40$ & Peru & -13.32 & -76.50 & 8.0 & ELTPCSP1 \\
\hline Sep 12 & $11: 10$ & Indonesia & -4.517 & 101.38 & 8.5 & ELTP1 \\
\hline Sep 28 & $13: 38$ & USA & 21.980 & 142.68 & 7.5 & EPV1 \\
\hline & $05: 23$ & New Zealand & -49.39 & 163.84 & & \\
\hline Oct 15 & $12: 29$ & New Zealand & -44.68 & 167.21 & 6.8 & EPV1 \\
\hline Oct 24 & 21:02 & Indonesia & -3.909 & 101.06 & 6.8 & ELTP2 \\
\hline Oct 31 & 03:04 & USA & 37.432 & -121.7 & 5.6 & ELTP1 \\
\hline Oct 31 & 03:30 & A & 18.854 & 145.31 & .2 & $\mathrm{P} 2$ \\
\hline Nov 14 & $15: 40$ & Chile & -22.18 & -69.84 & 7.7 & ELTPCSP \\
\hline Nov 25 & $16: 02$ & Indonesia & -8.294 & 118.36 & 6.5 & EPVD1 \\
\hline Nov 29 & 19:00 & France & 14.951 & -61.24 & 7.4 & EPV1 \\
\hline Dec 9 & $07: 28$ & Fiji & -26.15 & -177.4 & 7.8 & EVP1 \\
\hline Dec 19 & 09:30 & Alaska, USA & 51.495 & -179.4 & 7.1 & ELTP1 \\
\hline
\end{tabular}


2008

\begin{tabular}{|c|c|c|c|c|c|c|}
\hline Date & $\begin{array}{c}\text { Time } \\
\text { (UTC) }\end{array}$ & Place & Lat. & Long. & $\begin{array}{l}\text { Mag- } \\
\text { nitude }\end{array}$ & Comments \\
\hline $\operatorname{Jan} 5$ & 11:01 & Canada & 51.299 & -130.7 & 6.6 & ELTPCSP1 \\
\hline Feb 3 & 07:34 & Congo & -2.314 & 28.896 & 5.9 & EVP1 \\
\hline Feb 14 & 10:09 & Greece & 36.646 & 21.833 & 6.9 & ELTP2 \\
\hline Feb 20 & 08:08 & Indonesia & 2.751 & 95.966 & 7.4 & EPV1 \\
\hline Feb 21 & $15: 26$ & (Norway) & 77.41 & 14.48 & 6.1 & ELTP1 \\
\hline Feb 21 & $14: 16$ & USA & 41.076 & 114.77 & 6.0 & TPPTE1 \\
\hline Apr 18 & 09:37 & USA & 38.450 & 87.890 & 5.2 & ELTP1 \\
\hline May 7 & $16: 45$ & Japan & 36.141 & 141.54 & 6.9 & ELTP2 \\
\hline May 12 & $06: 28$ & China & 31.099 & 103.27 & 7.9 & ELTP2 \\
\hline May 24 & $19: 20$ & Colombia & 4.447 & -73.67 & 5.9 & EPV2 \\
\hline May 29 & $15: 46$ & Iceland & 63.992 & -21.01 & 6.3 & ELTP2 \\
\hline Jun 8 & $12: 25$ & Greece & 38.029 & 21.464 & 6.4 & TPPTE1 \\
\hline Jun 13 & $23: 43$ & Japan & 39.122 & 140.67 & 6.9 & ELTP2 \\
\hline Jul 15 & $03: 26$ & Greece & 35.983 & 27.785 & 6.4 & ELTP1 \\
\hline Jul 19 & 02:39 & Japan & 37.627 & 142.11 & 7.0 & SP1 \\
\hline Jul 23 & $15: 26$ & Japan & 39.807 & 141.46 & 6.8 & ELTP2 \\
\hline Jul 29 & $18: 42$ & California & 33.955 & -117.7 & 5.5 & ELTP2 \\
\hline Aug 21 & $12: 24$ & China & 25.066 & 97.737 & 6.0 & EPV2 \\
\hline Aug 25 & $13: 21$ & China & 30.893 & 83.614 & 6.7 & ELTP2 \\
\hline Aug 30 & 08:30 & China & 26.277 & 101.91 & 6.0 & EPVD2 \\
\hline Sep 8 & $18: 52$ & Vanuatu & -13.51 & 166.96 & 6.9 & ELTP1 \\
\hline Sep 10 & 11:00 & Iran & 26.823 & 55.825 & 6.1 & ELTP1 \\
\hline Sep 11 & 00:20 & Japan & 41.979 & 143.62 & 6.8 & EPV1 \\
\hline Sep 29 & $15: 19$ & $\begin{array}{c}\text { New } \\
\text { Zealand }\end{array}$ & -29.87 & -177.6 & 7.0 & \\
\hline Oct 5 & $15: 52$ & Kyrgyzstan & 39.515 & 73.768 & 6.6 & ELTP2 \\
\hline Oct 6 & $08: 30$ & China & 29.759 & 90.302 & 6.3 & ELTP1 \\
\hline Oct 11 & 09:06 & Russia & 43.271 & 46.262 & 5.8 & ELTP2 \\
\hline Oct 16 & $19: 41$ & Mexico & 14.443 & -92.42 & 6.7 & ELTP2 \\
\hline Oct 19 & $05: 10$ & Tonga & -21.85 & -173.8 & 6.9 & ELTP2 \\
\hline Oct 28 & 23:09 & Pakistan & 30.653 & 67.323 & 6.4 & ELTP1 \\
\hline Nov 16 & 17:02 & Indonesia & 1.290 & 122.10 & 7.3 & EPV1 \\
\hline Nov 24 & 09:02 & Okhotsk & 54.194 & 154.31 & 7.3 & EPV2 \\
\hline
\end{tabular}

2009

\begin{tabular}{ccccccc}
\hline Date & $\begin{array}{c}\text { Time } \\
\text { (UTC) }\end{array}$ & Place & Lat. & Long. & $\begin{array}{c}\text { Mag- } \\
\text { nitude }\end{array}$ & Comments \\
\hline Jan 3 & $19: 43$ & Indonesia & -0.510 & 132.78 & 7.6 & ELTPCSP1 \\
Jan 8 & $19: 21$ & Costa Rica & 10.197 & -84.15 & 6.1 & ELTPCSP2 \\
Jan 15 & $17: 49$ & Russia & 46.862 & 155.15 & 7.4 & EPV1 \\
Feb 11 & $17: 34$ & Indonesia & 3.902 & 126.40 & 7.2 & ELTP1 \\
Mar 19 & $18: 17$ & Tonga & -23.05 & -174.6 & 7.6 & ELTPCSP2 \\
Apr 6 & $01: 32$ & Italy & 42.334 & 13.334 & 6.3 & ELTPCSP2 \\
Apr 7 & $04: 23$ & Islands & 46.088 & 151.49 & 6.9 & ELTPCSP1 \\
Apr 16 & $21: 27$ & Afghanistan & 34.197 & 70.065 & 5.4 & ELTPCSP1 \\
\hline
\end{tabular}

\begin{tabular}{|c|c|c|c|c|c|c|}
\hline May 28 & $08: 24$ & Honduras & 16.730 & -86.20 & 7.3 & TPPTECSP1 \\
\hline Jul 9 & $11: 49$ & China & 25.619 & 101.08 & 5.7 & EPV2 \\
\hline Jul 15 & 09:22 & New Zealand & -45.75 & 166.58 & 7.8 & EPV1 \\
\hline Aug 9 & $10: 55$ & Japan & 33.144 & 138.04 & 7.1 & ELTP1 \\
\hline Aug 10 & $19: 55$ & Andaman & 14.013 & 92.923 & 7.5 & EPV2 \\
\hline Sep 2 & $07: 55$ & Indonesia & -78.09 & 107.25 & 7.0 & TPPTE1 \\
\hline Sep 12 & $20: 06$ & Venezuela & 10.70 & -67.92 & 6.3 & ELTP1 \\
\hline Sep 29 & $17: 48$ & Samoa & 15.509 & -172.0 & 8.1 & ELTP1 \\
\hline \multirow[t]{2}{*}{ Sep 30} & $10: 16$ & Indonesia & -0.725 & 99.856 & 7.6 & ELTP2 \\
\hline & $22: 03$ & & -13.05 & 166.18 & 7.6 & SP1 \\
\hline \multirow[t]{2}{*}{ Oct 7} & $22: 18$ & Vanuatu & -12.55 & 166.32 & 7.8 & SP1 \\
\hline & $23: 13$ & & -13.14 & 166.29 & 7.3 & ELTP1 \\
\hline Dec 19 & $13: 02$ & Taiwan & 23.763 & 121.68 & 6.4 & ELTP2 \\
\hline \multicolumn{7}{|l|}{2010} \\
\hline Date & $\begin{array}{c}\text { Time } \\
\text { (UTC) }\end{array}$ & Place & Lat. & Long. & $\begin{array}{l}\text { Mag- } \\
\text { nitude }\end{array}$ & Comments \\
\hline Jan 3 & $22: 36$ & Solomon & -8.912 & 157.30 & 7.2 & ELTP2 \\
\hline Jan 10 & $00: 27$ & California & 40.645 & -124.7 & 6.5 & EPV1 \\
\hline Jan 12 & $21: 53$ & Haiti & 18.451 & -72.44 & 7.0 & ELTPCSP1 \\
\hline Feb 26 & $20: 31$ & Japan & 23.472 & 123.71 & 7.0 & ELTPCSP1 \\
\hline Feb 27 & $03: 34$ & Chile & -35.84 & -72.71 & 8.8 & SP1 \\
\hline Feb 27 & $15: 45$ & Argentina & & & 6.1 & ELTPCSP1 \\
\hline Mar 4 & $0: 18$ & Taiwan & & & 6.4 & EPV1 \\
\hline Mar 5 & $23: 29$ & Sumatra & & & 6.5 & ELTP1 \\
\hline Mar 8 & $4: 32$ & Turkey & & & 6.0 & ELTP1 \\
\hline Mar 11 & $11: 39$ & Chile & -34.25 & -71.88 & 6.9 & ELTPCSP1 \\
\hline Mar 14 & $9: 57$ & Indonesia & & & 6.4 & EPV1 \\
\hline Mar 14 & $8: 08$ & Japan & & & 6.6 & ELTPCSP1 \\
\hline Mar 16 & $2: 21$ & Chile & & & 6.7 & EPV1 \\
\hline Apr 4 & $22: 40$ & Mexico & 2.128 & 115.3 & 7.2 & ELTP1 \\
\hline
\end{tabular}

\section{Analysis}

According to the information of 154 earthquakes over 6 scales of magnitude over the world from the USA Earthquake Observation Council, the causal relationship common area, symmetry of them we can get the following results, as shown in Table 2:

Table 2. Results.

\begin{tabular}{ccccccccc}
\hline Type & $\begin{array}{c}\text { Equatorial level } \\
\text { Line plane }\end{array}$ & $\begin{array}{c}\text { Which } \\
\text { Symmetry }\end{array}$ & $\begin{array}{c}\text { Ecliptic } \\
\text { Line plane }\end{array}$ & $\begin{array}{c}\text { Among } \\
\text { Symmetry }\end{array}$ & $\begin{array}{c}\text { Vertical } \\
\text { Flat }\end{array}$ & $\begin{array}{c}\text { Similar } \\
\text { point }\end{array}$ & $\begin{array}{c}\text { Other Not } \\
\text { coplanar }\end{array}$ & $\begin{array}{c}\text { Total } \\
\text { Quantity }\end{array}$ \\
$\begin{array}{c}\text { Percentage of total } \\
22\end{array}$ & $($ Four) & 77 & $(23)$ & 45 & 7 & 3 & 154 \\
\hline
\end{tabular}




\section{Conclusions}

This study allowed existence of the errors, but those errors do not interfere the co-planarity and the symmetry principle. Errors may come from the inaccuracy of observation time, perhaps the errors accelerate the process of the Earth becoming to sphere. In the next step of our research, we plan to study on earthquake prediction and the relationship between earthquake and economic development.

\section{References}

[1] Http://earthquake.usgs.gov/recenteqsww/quakes/quakes_ all.html 